\title{
New candidates for extremely metal-poor emission-line galaxies in the SDSS/BOSS DR10*
}

\author{
N. G. Guseva ${ }^{1,2}$, Y. I. Izotov ${ }^{1,2}$, K. J. Fricke ${ }^{1,3}$, and C. Henkel ${ }^{1,4}$ \\ ${ }^{1}$ Max-Planck-Institut für Radioastronomie, Auf dem Hügel 69, 53121 Bonn, Germany \\ e-mail: guseva@mao.kiev.ua \\ 2 Main Astronomical Observatory, Ukrainian National Academy of Sciences, Zabolotnoho 27, 03680 Kyiv, Ukraine \\ 3 Institut für Astrophysik, Göttingen Universität, Friedrich-Hund-Platz 1, 37077 Göttingen, Germany \\ 4 Astronomy Department, King Abdulaziz University, PO Box 80203, 21589 Jeddah, Saudi Arabia
}

Received 20 January 2015 / Accepted 30 April 2015

\begin{abstract}
We present a spectroscopic study of eight extremely low-metallicity candidate emission-line galaxies with oxygen abundances possibly below $12+\log \mathrm{O} / \mathrm{H}=7.35$. These galaxies were selected from data release 10 of the Sloan Digital Sky Survey/Baryon Oscillation Spectroscopic Survey (SDSS/BOSS DR10). We will call these extremely metal-deficient (XMD) galaxies. The electron temperaturesensitive emission line [O III] $\lambda 4363$ is detected in three galaxies and marginally detected in two galaxies, allowing for abundance determination by a "direct" method. Because of large uncertainties in the [O III] $\lambda 4363 \AA$ line fluxes, we also calculated oxygen abundance in these galaxies together with the remaining three galaxies using a strong-line semi-empirical method. This method gives oxygen abundances higher than 7.35 for three galaxies with detected [O III] $\lambda 4363 \AA$ line and lower than 7.35 for the remaining five objects of the sample. The newly-discovered galaxies represent excellent targets for follow-up spectroscopic observations with the largest telescopes to improve the oxygen abundance determination and to increase the number of these very rare low-metallicity objects. The extreme location of the most massive and luminous XMD galaxies and XMD candidates in the stellar mass-metallicity diagram implies that these galaxies may be genuine young objects. With stellar masses of up to $\sim 10^{7}-10^{8} M_{\odot}$, the galaxies are not chemically enriched and strongly deviate to lower metallicity as compared to the relation obtained for a large sample of low-redshift, star-forming galaxies.
\end{abstract}

Key words. galaxies: abundances - galaxies: irregular - galaxies: evolution - galaxies: formation - galaxies: ISM - ISM: abundances

\section{Introduction}

Searching for extremely metal-poor emission-line galaxies is very important for several reasons. One of them is that these galaxies are the most promising young galaxy candidates in the local Universe (Guseva et al. 2003; Izotov \& Thuan 2004b). In this paper we consider extremely metal-deficient (hereafter XMD) emission-line galaxies, searching for targets with oxygen abundances $12+\log \mathrm{O} / \mathrm{H} \leq 7.35$.

The physical conditions in the interstellar medium (ISM) of XMDs provide insights into a variety of aspects of fundamental physics, cosmology, and astronomy. These galaxies have very low, but not pristine element abundances, hinting at preenrichment by e.g. Population III stars (Thuan et al. 2005). Large enough statistics is needed to confirm this idea. The galaxies with very low metallicity share many properties of Ly $\alpha$ emitting galaxies and Ly-break galaxies (Prochaska et al. 2003; Nakajima et al. 2013; Shirazi et al. 2014; Shapley et al. 2015). Thanks to the fact that XMDs can be excellent local proxies for their high- $z$ counterparts. These chemically unevolved galaxies are also the best objects for the determination of the primordial ${ }^{4} \mathrm{He}$ abundance $Y_{\mathrm{p}}$ (e.g. Izotov \& Thuan 2004a, 2010; Izotov et al. 2007, $2013,2014 a)$. A more detailed review of their properties can be found in Izotov et al. (2012).

* Tables 2-4 are available in electronic form at http://www . aanda.org
Many efforts were undertaken recently to search for these very rare objects (see e.g. Pustilnik et al. 2005; Izotov et al. 2006b; Izotov \& Thuan 2007, 2009; Guseva et al. 2007; Pustilnik et al. 2011; Izotov et al. 2012; Berg et al. 2012). More than ten galaxies with $12+\log \mathrm{O} / \mathrm{H} \leq 7.35$ were found among the galaxies from the Sloan Digital Sky Survey data release 7 (SDSS DR7; Izotov et al. 2012). To further increase the number of these objects, we searched for new XMD candidates among the objects from the extention of the SDSS survey, SDSS-III, namely among the galaxies that appeared in the SDSS/BOSS data release 10 (DR10).

The data and the element abundance determination are described in Sect. 2. The results are presented in Sect. 3. More specifically, we consider XMD emission-line galaxies with respect to the luminosity-metallicity relations in Sect. 3.1, their location in the emission-line diagnostic diagram in Sect. 3.2, and in the mass-metallicity diagram in Sect. 3.3. Our main conclusions are summarised in Sect. 4.

\section{The data}

We use a sample of $~ 9000$ star-forming galaxies by Izotov et al. (2015) selected from the spectroscopic database of the SDSS/BOSS DR10 (Ahn et al. 2014). The details of data selection can be found in Izotov et al. (2014a). Out of this large sample, we could only identify eight candidates that may represent extremely low-metallicity emission-line galaxies with 
Table 1. General characteristics of newly identified XMD candidates.

\begin{tabular}{|c|c|c|c|c|c|c|c|c|}
\hline \multirow[t]{2}{*}{ Name } & \multirow[t]{2}{*}{$\overline{\mathrm{RA}(\mathrm{J} 2000.0)^{a}}$} & \multirow[t]{2}{*}{$\operatorname{Dec}(\mathrm{J} 2000.0)^{a}$} & \multirow[t]{2}{*}{ Redshift } & \multirow[t]{2}{*}{$g^{b}$} & \multirow[t]{2}{*}{$M_{\mathrm{g}}{ }^{c}$} & \multirow[t]{2}{*}{$M_{\text {stars }}{ }^{d}$} & \multicolumn{2}{|c|}{$12+\log \mathrm{O} / \mathrm{H}^{e}$} \\
\hline & & & & & & & $\mathrm{T}_{e}$-method & strong-line method \\
\hline $\mathrm{J} 0100-0028$ & 01:00:56.93 & $-00: 28: 43.9$ & 0.0192 & 20.85 & -14.43 & 6.62 & & 7.10 \\
\hline $\mathrm{J} 0122+0048$ & $01: 22: 41.61$ & $+00: 48: 42.0$ & 0.0574 & 21.62 & -16.16 & 6.45 & $7.22 \pm 0.15$ & 7.35 \\
\hline $\mathrm{J} 0143+1958^{f}$ & $01: 43: 15.15$ & $+19: 58: 06.1$ & 0.0017 & 21.76 & -7.66 & 4.28 & & 7.28 \\
\hline J0153+0104 & 01:53:11.96 & $+01: 04: 40.1$ & 0.0632 & 21.51 & -16.23 & 6.34 & $7.34 \pm 0.12$ & 7.60 \\
\hline J0222-0935 & $02: 22: 38.55$ & $-09: 35: 35.2$ & 0.1148 & 21.61 & -17.30 & 8.04 & $7.29 \pm 0.32$ & 7.43 \\
\hline J0945+3835 & 09:45:19.55 & $+38: 35: 52.9$ & 0.0723 & 21.78 & -16.82 & 7.24 & $7.33 \pm 0.20$ & 7.48 \\
\hline $\mathrm{J} 1036+2036$ & 10:36:39.47 & $+20: 36: 15.8$ & 0.0549 & 21.59 & -15.61 & 6.82 & $7.32 \pm 0.34$ & 7.35 \\
\hline J1228-0125 & $12: 28: 45.54$ & $-01: 25: 26.9$ & 0.0728 & 22.09 & -15.58 & 6.99 & $\ldots$ & 7.33 \\
\hline
\end{tabular}

Notes. ${ }^{(a)}$ Equatorial coordinates. ${ }^{(b)}$ SDSS $g$ magnitude. ${ }^{(c)}$ Absolute $g$ magnitude. ${ }^{(d)}$ Log of stellar mass in unit of solar mass. ${ }^{(e)}$ This paper. (f) $\mathrm{J} 0143+1958$ is part of galaxy UGCA 20 .
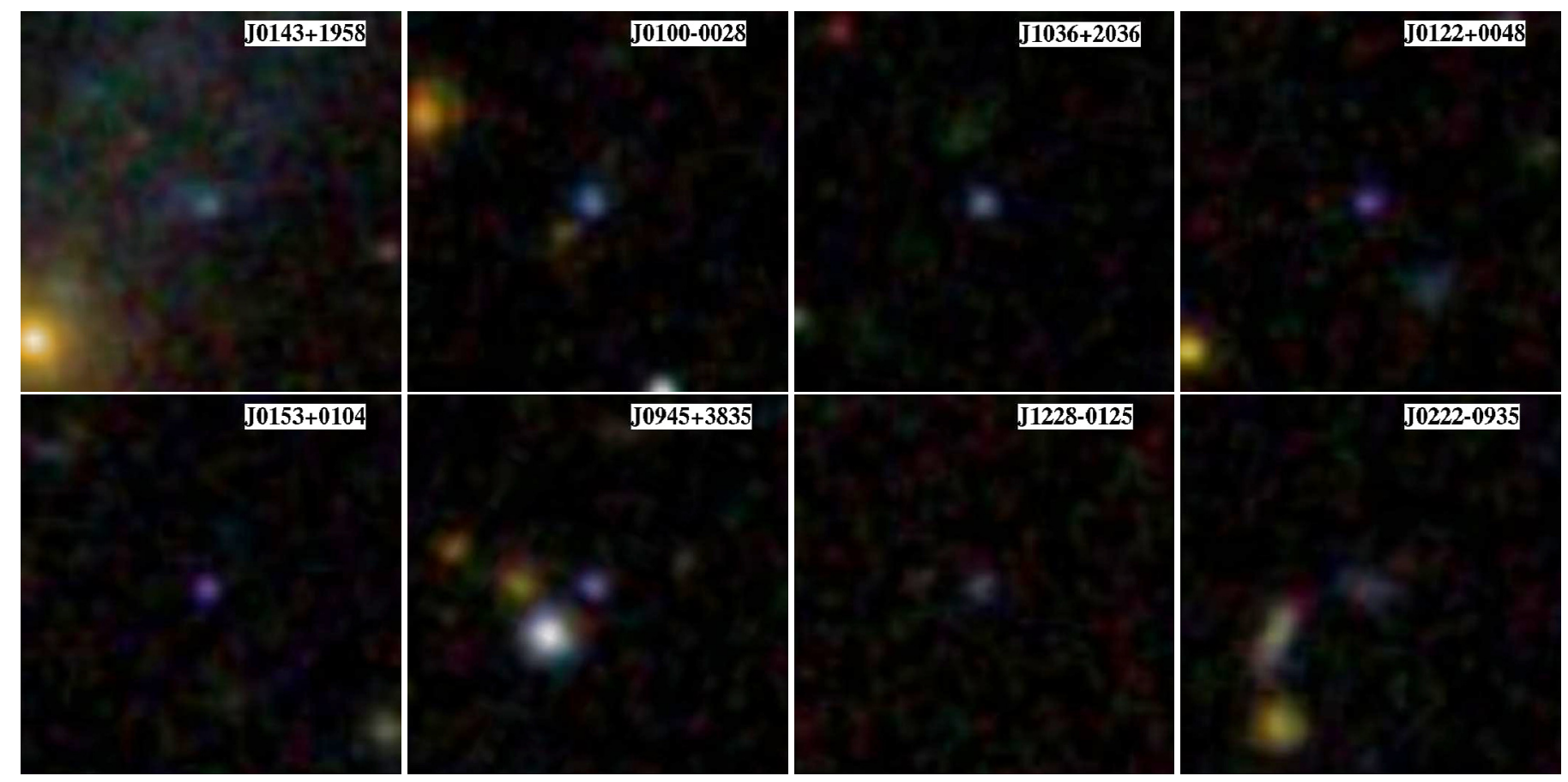

Fig. 1. $2^{\prime} \times 2^{\prime}$ SDSS images of the galaxies ordered according to increasing redshift.

$12+\log \mathrm{O} / \mathrm{H} \leq 7.35$. The general characteristics of these galaxies, such as the name of galaxy, coordinates, redshift, apparent $g$ magnitude, absolute $g$ magnitude, and stellar mass are collected in Table 1. The last two columns of the table show oxygen abundances we derived using two methods (see below). SDSS images of the galaxies with labelled names and ordered following increasing redshift are shown in Fig. 1. The rest-frame SDSS spectra of the galaxies arranged with increasing redshift are presented in Fig. 2.

We measured the line fluxes and their errors using the IRAF ${ }^{1}$ SPLOT routine. The line flux errors are later propagated into the calculation of abundance errors. We derived the internal extinction from the Balmer hydrogen emission lines after correction for the Milky Way extinction. The line fluxes were corrected for both reddening (Whitford 1958) and underlying hydrogen stellar absorption by the application of an iterative procedure (Izotov et al. 1994). The observed line intensities $F(\lambda) / F(\mathrm{H} \beta)$, the extinction-corrected line intensities $I(\lambda) / I(\mathrm{H} \beta)$, multiplied

IRAF is the Image Reduction and Analysis Facility distributed by the National Optical Astronomy Observatory, which is operated by the Association of Universities for Research in Astronomy (AURA) under cooperative agreement with the National Science Foundation (NSF). by 100 , and equivalent widths of emission lines are shown in Table 2 together with extinction coefficients $C(\mathrm{H} \beta)$, observed $\mathrm{H} \beta$ fluxes, and equivalent widths $E W(\mathrm{abs})$ of the hydrogen absorption stellar lines.

Finally, we selected the five XMD candidates with the detected [O III] $\lambda 4363 \AA$ emission line. This allows for a reliable oxygen abundance determination using the direct $T_{\mathrm{e}}$-method and following the prescriptions of Izotov et al. $(1994,1997)$ and Thuan et al. (1995). The temperature $T_{\mathrm{e}}(\mathrm{O}$ III) is calculated based on the $[\mathrm{O}$ III] $\lambda 4363 /(\lambda 4959+\lambda 5007)$ line ratio. For the remaining three XMD candidates the semi-empirical method by Izotov \& Thuan (2007) was applied to derive $T_{\mathrm{e}}(\mathrm{O}$ III). We adopt a two-zone photoionised $\mathrm{H}$ II region model: a high-ionisation zone with temperature $T_{\mathrm{e}}(\mathrm{O}$ III $)$, where [O III] and [Ne III] lines originate, and a low-ionisation zone with temperature $T_{\mathrm{e}}(\mathrm{O}$ II $)$, where [O II] lines originate. For $T_{\mathrm{e}}(\mathrm{O}$ II $)$, we use the relation between the electron temperatures $T_{\mathrm{e}}(\mathrm{O}$ III $)$ and $T_{\mathrm{e}}(\mathrm{O}$ II $)$ obtained by Izotov et al. (2006a) from the $\mathrm{H}$ II photoionisation models of Stasińska \& Izotov (2003).

We derived ionic and total oxygen and neon abundances using expressions for ionic abundances and the ionisation correction factor for neon by Izotov et al. (2006a). In Table 3 
N. G. Guseva et al.: New XMD galaxies in SDSS/BOSS DR10

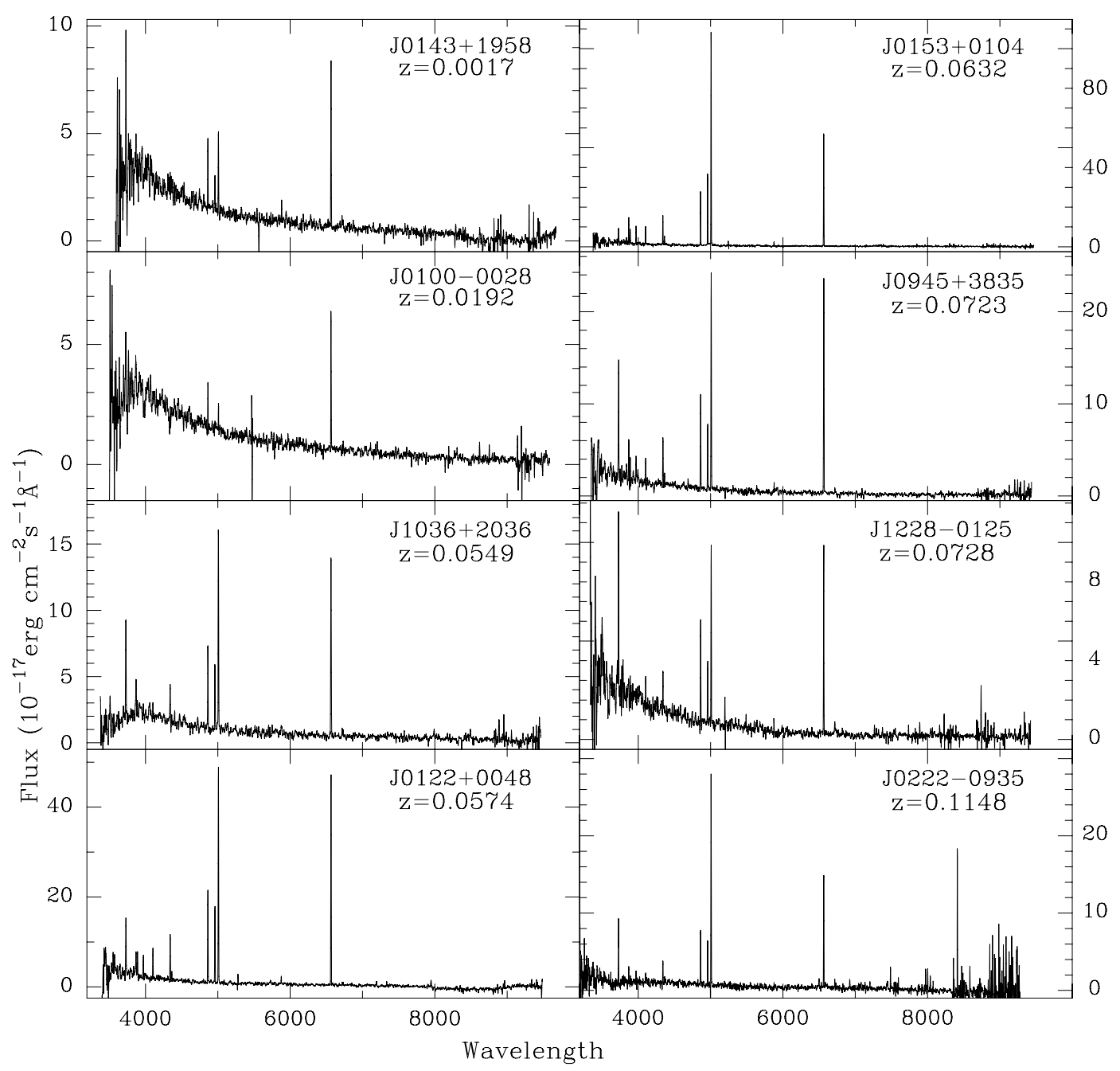

Fig. 2. SDSS spectra of the eight extremely metal-deficient galaxies.

the electron temperatures $T_{\mathrm{e}}\left(\mathrm{O}\right.$ II) and $T_{\mathrm{e}}(\mathrm{O}$ III $)$, and oxygen and neon abundances are given for three galaxies with detected and for two galaxies with marginally detected [O III]4363 line. All five XMD candidates have very low oxygen abundances $12+\log \mathrm{O} / \mathrm{H}<7.35$ (Table 3 ). The oxygen abundance in the remaining three galaxies with undetected [O III]4363 lines is derived with the strong-line semi-empirical method (Table 4). These three galaxies also have very low oxygen abundances $12+\log \mathrm{O} / \mathrm{H}<7.35$.

Because of large uncertainties in the $[\mathrm{O}$ III] $] 4363 \AA$ line fluxes, we also recalculated oxygen abundance for the five galaxies with detected and marginally detected [O III] $\lambda 4363 \AA$ (Table 3) using a strong-line semi-empirical method. This method gives oxygen abundances higher than 7.35 for three galaxies with detected $\lambda 4363 \AA$ line (see Table 4$)$. The largest difference is found for the galaxy J0153+0104 with the best derived flux of the $[\mathrm{O}$ III] $\lambda 4363 \AA$ line and the best determination of the oxygen abundance with the $T_{\mathrm{e}}$-method. This fact indicates that strong-line methods may not be used for accurate abundance determination. Instead higher-quality observations are needed to improve oxygen abundances in the considered galaxies. We do not show the sulfur abundances derived by using [S III] $\lambda 9069$ and $\lambda 9531$ in Tables 3 and 4 . These lines are observed in noisy parts of the spectra with numerous residuals of night sky lines, making sulfur abundances uncertain.

\section{Results}

In this section, we discuss the oxygen abundances and the distributions of the extremely metal-deficient galaxies in the broad-band luminosity-metallicity, $\mathrm{H} \beta$ luminosity-metallicity, diagnostic Baldwin-Phillips-Terlevich, and mass-metallicity diagrams. Throughout this Section we compare the XMD candidates with previous samples of similar emission-line galaxies, analysing all these data in a uniform way (Izotov et al. 2006a, 2011, 2012, 2014a; Izotov \& Thuan 2007).

It is seen from Tables 3 and 4 that no galaxy with an oxygen abundance $12+\log \mathrm{O} / \mathrm{H}<7.1$ was found. A similar conclusion was obtained in previous studies (e.g. Izotov et al. 2012). This supports the idea discussed by, e.g., Thuan et al. (2005) that the matter from which dwarf emission-line galaxies were formed was pre-enriched to a level $12+\log \mathrm{O} / \mathrm{H} \gtrsim 6.9$ (or $2 \%$ of the abundance $12+\log \mathrm{O} / \mathrm{H}=8.69$ of the Sun, Asplund et al. 2009). They also showed that BCDs spanning a wide range in ionised gas metallicities all have $\mathrm{H}$ I envelopes with about the same neutral gas metallicity of $\sim 7.0$. This is also the metallicity found in Ly $\alpha$ absorbers (Prochaska et al. 2003). Taken together, the available data suggest that the primordial neutral gas may have been previously enriched to a common metallicity level $12+\log \mathrm{O} / \mathrm{H} \sim 6.9-7.0$, possibly by Population III stars.

The number of XMDs with $12+\log \mathrm{O} / \mathrm{H} \leq 7.35$ is yet very small. Only eight XMD candidates out of $\sim 9000$ emission-line 

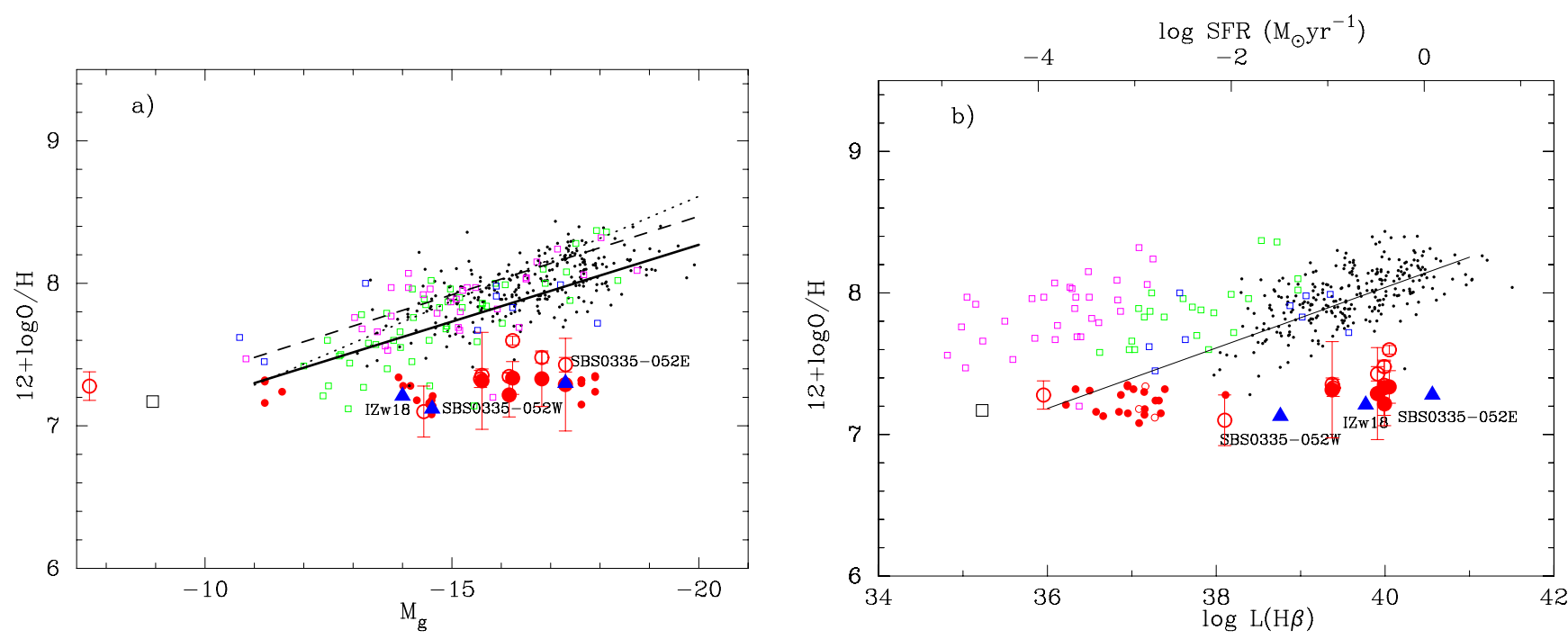

Fig. 3. Panel a) absolute SDSS $g$ magnitude-oxygen abundance relation for the five galaxies with oxygen abundances derived using the "direct" method (large red filled circles) and for all eight galaxies with oxygen abundances derived with the semi-empirical method by Izotov \& Thuan (2007) (large red open circles). We also show the XMD emission-line galaxies with $12+\log \mathrm{O} / \mathrm{H} \leq 7.35$ by Izotov \& Thuan (2007) and Izotov et al. (2012) in small red filled circles. The small open squares in magenta, green, and blue are the data derived by Berg et al. (2012), Pustilnik et al. (2011), and James et al. (2015), respectively (see text). The most-metal deficient BCDs I Zw 18, SBS 0335-052W, and SBS 0335-052E are shown with filled blue triangles and are labelled. One of the most metal-poor dwarf galaxies, Leo P from Skillman et al. (2013) is plotted with a large open black square. For comparison, the "standard" $L-Z$ relation for emission-line galaxies (SDSS and star-forming galaxies from Izotov et al. 2006a; Izotov \& Thuan 2007) are shown in black dots with the linear regression to these data (solid line). The relations derived by Richer \& McCall (1995) and by Berg et al. (2012) are shown in a dotted line and a dashed line, respectively. Panel b) extinction-corrected H $\beta$ luminosity-oxygen abundance relation. The samples and symbols are the same as in panel a). The solid line represents the linear regression to SDSS galaxies (Izotov et al. 2006a) and to all galaxies from Izotov \& Thuan (2007), excluding I Zw 18, SBS 0335-052W, and SBS 0335-052E, and to galaxies from Izotov et al. (2012).

galaxies extracted from SDSS DR 10 , or $<0.1 \%$, were found, indicating that these objects are very scarce in the Local Universe.

\subsection{Luminosity-metallicity relations}

The existence of the relation between optical luminosities and metallicities of galaxies, the so called $L-Z$ relation, was established by Lequeux et al. (1979) and later confirmed by Skillman et al. (1989) and Richer \& McCall (1995). The metallicity of a galaxy is a measure of the abundance of metals relative to hydrogen in the ISM and is most commonly quoted in terms of the oxygen abundance $12+\log \mathrm{O} / \mathrm{H}$. As a measure of the optical luminosity, we use the $M_{\mathrm{g}}$ absolute magnitude obtained from the apparent SDSS $g$ magnitudes (Table 1), at variance with early studies of the $L-Z$ relation, in what the apparent $B$ magnitude was commonly used. However, Papaderos et al. (2008) have shown that SDSS $g$ magnitudes are very similar to $B$ magnitudes of star-forming galaxies. The distances to the galaxies are derived from their radial velocities, adopting a Hubble constant $H_{0}=70 \mathrm{~km} \mathrm{~s}^{-1} \mathrm{Mpc}^{-1}$.

In Fig. 3a the absolute $g$ magnitude-metallicity relation for SDSS galaxies by Izotov et al. (2006a), as a representative sample, is plotted in black dots. We want to examine how much the XMDs differ from the star-forming galaxies in "standard" samples. The solid line represents the linear regression to SDSS galaxies (Izotov et al. 2006a) and to all galaxies from Izotov \& Thuan (2007). The position of all XMD candidates from this paper and from Izotov \& Thuan (2007) and Izotov et al. (2012) are also plotted.

Berg et al. (2012) studied a sample of 42 low-luminosity galaxies aiming to fill the relatively sparse low-luminosity end of the relation with intrinsically faint galaxies. For comparison we show their data in small open squares in magenta. Aiming to find the low-metallicity emission-line galaxies in the voids Pustilnik et al. (2011) discovered some objects with low metallicity, which are plotted in Fig. 3 with green open squares. Blue diffuse dwarf (BDD) galaxies with star-formation activity from James et al. (2015) are shown with open blue squares. The most-metaldeficient BCDs I Zw 18, SBS 0335-052W, and SBS 0335-052E are shown in filled blue triangles and are labelled. One of the most metal-deficient star-forming nearby dwarf galaxies, Leo $\mathrm{P}$ (Skillman et al. 2013) is plotted with a large open black square. We also plotted the relation derived by Richer \& McCall (1995) (dotted line) and the relation of Berg et al. (2012) (dashed line), which are not very different from the relation by Izotov \& Thuan (2007).

Most XMDs occupy the same region in the $L-Z$ diagram as the well-known metal-deficient BCDs I Zw 18 and SBS 0335052E. All these galaxies are systematically shifted to lower metallicities relative to the standard line for every bin of the $M_{\mathrm{g}}$ range from -12 to -18 mag. Considering a higher oxygen abundance cutoff $12+\log (\mathrm{O} / \mathrm{H}) \leq 7.65$ Kniazev et al. (2003) and Guseva et al. (2009) found a large scatter of galaxies filling the gap between the solid line in Fig. 3a and the location of XMDs with $12+\log (\mathrm{O} / \mathrm{H})<7.35$.

Several possible evolutionary scenarios were suggested for these galaxies. One of them implies that they are genuine chemically unevolved (or young) galaxies with low metallicity. At $M_{\mathrm{g}} \sim-17$ they are shifted down by about 0.5-0.6 dex relative to the standard $L-Z$ line. This implies that about $0.09 \%$ of quite typical emission-line galaxies, populating the Local Universe, show a sizable deficiency of oxygen, amounting to not more than $2-4 \%$ of the metallicity compared to the bulk of galaxies used for producing the $L-Z$ relation. 
A likely alternative interpretation of the observed shift is an increase in luminosity at a given metallicity because of strong SF activity (e.g. Guseva et al. 2009). Most extreme XMD outliers are shifted from the "standard" $L-Z$ relation (represented, for instance, with the relation of Richer \& McCall 1995) by more than 6-7 mags (Fig. 3a). Including most-metal deficient galaxies, especially those with strong and very strong ongoing star formation, leads to a shallower slope of the $L-M$ relation (Guseva et al. 2009). In particular, XMDs with $12+\log (\mathrm{O} / \mathrm{H})<$ 7.35 and XMD candidates do not show a dependence on metallicity at all.

In Fig. 3b, the diagram $\mathrm{H} \beta$ luminosity $L(\mathrm{H} \beta)$ metallicity is plotted for the same galaxies as in Fig. 3a. The upper axis is a scale for the star formation rate (SFR), derived from the $\mathrm{H} \alpha$ luminosity as defined by Kennicutt (1998). As seen in Fig. 3b, XMDs have a large spread of $\mathrm{H} \beta$ luminosity from $10^{36}$ to $10^{40} \mathrm{erg} \mathrm{s}^{-1}$. Six out of eight galaxies from our new XMD candidate sample are characterised by strong SF activity, which results in the presence of high-excitation $\mathrm{H}$ II regions with strong emission lines. Their SFRs are in the range $\sim 0.01-0.5 M_{\odot} \mathrm{yr}^{-1}$ and are close to the SFRs of I Zw 18 and the SBS 0335-052 system. Only one object from our newly discovered XMDs, $\mathrm{J} 0143+1958$, has an $\mathrm{H} \beta$ luminosity corresponding to one late $\mathrm{O}$ or early B star assuming that a single O5V star produces the $\mathrm{H} \beta$ luminosity of $4.8 \times 10^{36} \mathrm{erg} \mathrm{s}^{-1}$ (leftmost outlying open circles in Figs. 3a and b, Leitherer 1990). This is in fact not a galaxy, but an H II region in the nearby low-surface-brightness dwarf galaxy UGCA 20, while our other XMDs are more distant compact galaxies with spectra, which are representative for the entire galaxy.

The galaxies by Berg et al. (2012) denoted by small open magenta squares in Fig. 3a have $\log L(\mathrm{H} \beta)$ in the range of 3537 and belong to galaxies with low-excitation $\mathrm{H}$ II regions and higher metallicity as compared to our sample of XMD galaxies and XMD candidates.

Thus, intense starbursts with high SFRs, similarly to the well-known BCDs I Zw 18 and SBS 0335-052E (Sargent \& Searle 1970; Izotov et al. 1990), were discovered in six out of eight XMD candidates.

\subsection{The emission-line diagnostic diagram}

In Fig. 4 we plot the location of XMDs in the Baldwin-PhillipsTerlevich (BPT) diagnostic diagram (Baldwin et al. 1981). As seen in the figure, the newly discovered most metal-poor galaxies are located at the same place as the XMDs with $12+$ $\log \mathrm{O} / \mathrm{H} \leq 7.35$ collected by Izotov et al. (2012). All these galaxies are far below the region, where the luminous compact galaxies (LCGs) by Izotov et al. (2011) are located (black dots). Solid and dashed lines are the lines by Stasińska et al. (2006) and Kauffmann et al. (2003), respectively, separating star-forming galaxies and AGNs. Similar to Fig. 3 the location of most newly discovered XMD candidates in Fig. 4 is close to that of the most metal-deficient BCDs I Zw 18 and SBS 0335-052E+W. The XMDs presently known populate a region that is so far almost empty. Thus, our discovery of these very scarce objects in the Local Universe appreciably increases the statistics needed to understand the nature of these galaxies.

\subsection{Mass-metallicity relation}

The relation between the stellar mass and its metallicity is a fundamental relation linking these two global galaxy properties. Mass is more fundamentally related to metallicity than luminosity. The luminosity of a galaxy is enhanced (active phase) or not

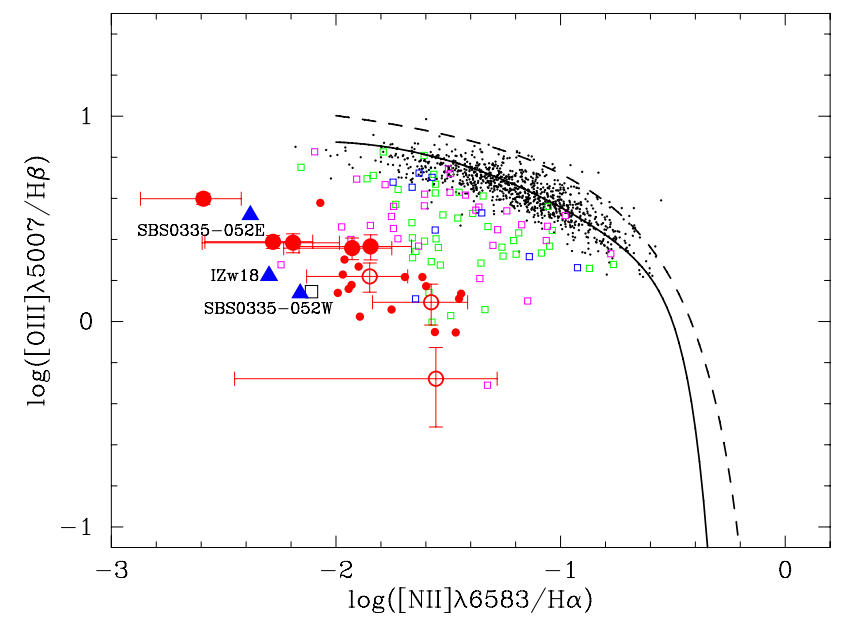

Fig. 4. Diagnostic diagram (BPT, Baldwin et al. 1981) for the XMD emission-line galaxies with $12+\log \mathrm{O} / \mathrm{H} \leq 7.35$ and our XMD candidates. Symbols are the same as in Fig. 3. We also plot a sample of 803 luminous compact galaxies (LCGs, black dots, Izotov et al. 2011). The dashed line from Kauffmann et al. (2003) and the solid line from Stasińska et al. (2006) separate star-forming galaxies (left side) from active galactic nuclei (right side).

enhanced (passive phase) by ongoing star formation, while stellar mass and metallicity are both determined by a galaxy's evolution in the past. We show our XMD candidates on the stellar mass-metallicity $\left(M_{*}-Z\right)$ diagram (Fig. 5). The solid line is the $M_{*}-Z$ relation obtained by Izotov et al. (2014a) for SDSS DR7 emission-line galaxies with robust metallicity determinations (black dots). For these galaxies, the errors of the [O III] 4363 emission-line fluxes do not exceed $25 \%$.

To derive stellar masses we modelled spectral energy distributions (SEDs) in the entire spectral range of $\lambda \lambda 3600$ $10300 \AA$, and subtracted gaseous line and continuum emission. The method is based on fitting a series of model SEDs to the observed SED and finding the best fit (Guseva et al. 2006, 2007; Izotov et al. 2011). Star formation history was approximated by a recent short burst in ages $<10 \mathrm{Myr}$, which accounts for the young stellar population, and a prior continuous star formation responsible for older stars of ages, that vary between $10 \mathrm{Myr}$ and 15 Gyr.

Our eight XMD candidates are shown in large red circles. All of them, except the low-mass H II region in UGCA 20, are located in the same area, as I Zw 18 and SBS 0335-052E. They are shifted by several orders of magnitude to high stellar masses as compared to stellar masses defined by the $M_{*}-Z$ relation for SDSS DR7 emission-line galaxies. For the range of stellar masses of these galaxies of $\sim 2 \times 10^{6}-10^{8} M_{\odot}$, their oxygen abundances are 3-4 times lower than predicted from the $M_{*}-Z$ relation for the SDSS DR7 emission-line galaxy sample. These differences may likely be due to the relatively young ages of XMDs, which had not enough time to evolve to higher metallicities, and support the idea that these least chemically evolved galaxies are genuine young galaxies. Many XMD galaxies are interacting systems (Ekta et al. 2008). Ekta \& Chengalur (2010) proposed an alternative hypothesis that XMD galaxies deviate from the $L-Z$ and $M_{*}-Z$ relations because of a combination of having low effective chemical yields and higher gas fractions due to tidal interactions.

Presently, XMDs are characterised by very high specific star formation rates (sSFR) of 10-100 $\mathrm{Gyr}^{-1}$. These high sSFRs contradict the conclusion by Shi et al. (2014) that the star formation efficiences in dwarf low-metallicity galaxies are less than a tenth 


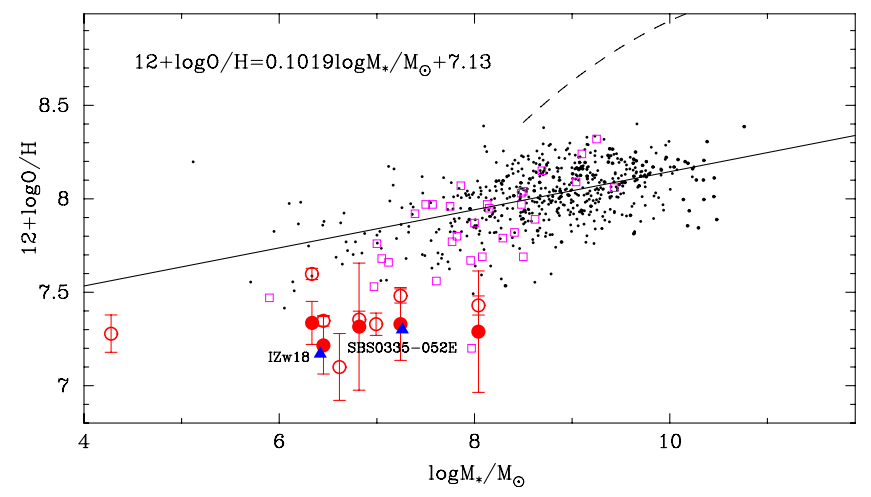

Fig. 5. Stellar mass oxygen abundance relation. Emission-line XMD galaxies with $12+\log \mathrm{O} / \mathrm{H}<7.35$ and our XMD candidates are shown in red and blue. Symbols are the same as in Fig. 3. For comparison, the SDSS DR7 emission-line galaxy sample by Izotov et al. (2014a), where the errors in [O III] $\lambda 4363$ emission-line flux do not exceed $25 \%$, are shown in black dots. The linear likelihood regression for this SDSS DR7 sample is plotted with a solid line. The small open squares in magenta are the data derived by Berg et al. (2012). The mass-metallicity relation obtained from 53000 SDSS star-forming galaxies by Tremonti et al. (2004) is plotted with a dashed line.

of the one found in normal metal-rich galaxies and that therefore star formation may have been inefficient in the early Universe.

\section{Conclusions}

We present a spectroscopic study of eight candidates for extremely metal-deficient (XMD) emission-line galaxies selected from the Sloan Digital Sky Survey/Baryon Oscillation Spectroscopic Survey data release 10 (SDSS/BOSS DR10), a part of the SDSS-III survey. We merged this sample with analogous XMD galaxies from the SDSS DR7 (Izotov et al. 2012) and from previous investigations. Our main results are as follows.

We find that oxygen abundances in all eight galaxies are very low and classify them as XMD candidates. The oxygen abundances in five XMD candidates with detected or marginally detected [O III] $\lambda 4363$ emission line were derived by the "direct" method. Note, however, the large uncertainties in the weak [O III] $\lambda 4363$ line in some galaxies. Six out of eight newly discovered XMD candidates are characterised by strong SF activity and high SFRs, similar to those in the well-known BCDs I Zw 18 and SBS 0335-052E (Sargent \& Searle 1970; Izotov et al. 1990).

No emission-line galaxies with $12+\log \mathrm{O} / \mathrm{H} \sim 7.0$ were found in the entire combined SDSS DR7 and DR10 surveys and among other galaxies collected from the literature (this paper and Izotov et al. 2012). This finding supports conclusions by Thuan et al. (2005) and Izotov et al. (2012) that the matter, from which XMDs were formed, was probably pre-enriched to this level prior to their formation.

The XMDs with strong SF activity are the most prominent outliers in the luminosity-metallicity $(L-Z)$ and $\mathrm{H} \beta$ luminositymetallicity $(L(\mathrm{H} \beta)-Z)$ relations, increasing the dispersion in the low-metallicity end of the relations.

Extreme positions of the most massive and luminous XMDs in the mass-metallicity diagrams imply that the galaxies could be genuinely young. These galaxies with stellar masses in the range of $\sim 10^{7}-10^{8} M_{\odot}$ are not yet chemically enriched.
Acknowledgements. Y.I.I. and N.G.G. thank the hospitality of the Max-Planck Institute for Radioastronomy, Bonn, Germany. Funding for the Sloan Digital Sky Survey (SDSS-III) has been provided by the Alfred P. Sloan Foundation, the Participating Institutions, the National Science Foundation, the U.S. Department of Energy, the National Aeronautics and Space Administration, the Japanese Monbukagakusho, and the Max Planck Society, and the Higher Education Funding Council for England.

\section{References}

Ahn, C. P., Rachael, A., Prieto, A., et al. 2014, ApJS, 211, 17

Asplund, M., Grevesse, N., \& Sauval, A. J., \& Scott, P. 2009, ARA\&A, 47, 481 Baldwin, J. A., Phillips, M. M., \& Terlevich, R. 1981, PASP, 93, 5 Berg, D. A., Skillman, E. D., Marble, A. R., et al. 2012, ApJ, 754, 98 Ekta, B., \& Chengalur, J. N. 2010, MNRAS, 406, 1238

Ekta, B., Chengalur, J. N., Pustilnik, S. A. 2008, MNRAS, 391, 881 Guseva, N. G., Papaderos, P., Izotov, Y. I., et al. 2003, A\&A, 407, 105 Guseva, N. G., Izotov, Y. I., \& Thuan, T. X. 2006, ApJ, 644, 890

Guseva, N. G., Izotov, Y. I., Papaderos, P., \& Fricke, K. J. 2007, A\&A, 464, 885 Guseva, N. G., Papaderos, P., Meyer, H., Izotov, Y. I., \& Fricke, K. J. 2009, A\&A, 505,63

Izotov, Y. I., \& Thuan, T. X. 2004a, ApJ, 602, 200

Izotov, Y. I., \& Thuan, T. X. 2004b, ApJ, 616, 768

Izotov, Y. I., \& Thuan, T. X. 2007, ApJ, 665, 1115

Izotov, Y. I., \& Thuan, T. X. 2009, ApJ, 690, 1797

Izotov, Y. I., \& Thuan, T. X. 2010, ApJ, 710, L67

Izotov, Y. I., Guseva, N. G., Lipovetsky, V. A., Kniazev, A. Y., \& Stepanian, J. A. 1990, Nature, 343, 238

Izotov, Y. I., Thuan, T. X., \& Lipovetsky, V. A. 1994, ApJ, 435, 647

Izotov, Y. I., Thuan, T. X., \& Lipovetsky, V. A. 1997, ApJS, 108, 1

Izotov, Y. I., Stasińska, G., Meynet, G., Guseva, N. G., \& Thuan, T. X. 2006a, A\&A, 448, 955

Izotov, Y. I., Papaderos, P., Guseva, N. G., Fricke, K. J., \& Thuan, T. X. 2006b, A\&A, 454, 137

Izotov, Y. I., Thuan, T. X., \& Stasińska, G. 2007, ApJ, 662, 15

Izotov, Y. I., Guseva, N. G., \& Thuan, T. X. 2011, ApJ, 728, 161

Izotov, Y. I., Thuan, T. X., \& Guseva, N. G. 2012, A\&A, 546, A122

Izotov, Y. I., Stasinska, G., Guseva, N. G. 2013, A\&A, 558, A57

Izotov, Y. I., Guseva, N. G., Fricke, K. J., \& Henkel, C. 2014a, A\&A, 561, A33

Izotov, Y. I., Thuan, T. X., \& Guseva, N. G. 2014b, MNRAS, 445, 778

Izotov, Y. I., Guseva, N. G., Fricke, K. J., \& Henkel, C. 2015, MNRAS, in press [arXiv: 1505.05853]

James, B. L., Koposov, S., Stark, D. P., et al. 2015, MNRAS, 448, 2687

Kauffmann, G., Heckman, T. M., Tremonti, C., et al. 2003, MNRAS, 346, 1055

Kennicutt, R. C., Jr. 1998, ARA\&A, 36, 189

Kniazev, A. Y., Grebel, E. K., Hao, L., et al. 2003, ApJ, 593, 73

Leitherer, C. 1990, ApJS, 73, 1

Lequeux, J., Rayo, J. F., Serrano, A., Peimbert, M., \& Torres-Peimbert, S. 1979, A\&A, 80, 155

Nakajima, K., Ouchi, M., Shimasaku, K., et al. 2013, ApJ, 769, 3

Papaderos, P., Guseva, N. G., Izotov, Y. I., \& Fricke, K. J. 2008, A\&A, 491, 113

Prochaska, J. X., Gawiser, E., Wolfe, A. M., Castro, S., \& Djorgovski, S. G. 2003, ApJ, 595, L9

Pustilnik, S. A., Kniazev, A. Y., \& Pramskij, A. G. 2005, A\&A, 443, 91

Pustilnik, S. A., Teplyakova, A. L., Kniazev, A. Y. 2011, Astrophys. Bull., 66, 255

Richer, M. G., \& McCall, M. L. 1995, ApJ, 445, 642

Sargent, W. L. W., \& Searle, L. 1970, ApJ, 162, L155

Shapley, A. E., Reddy, N. A., Kriek, M., et al. 2015, ApJ, 801, 88

Shi, Y., Armus, L., Helou, G., et al. 2014, Nature, 514, 335

Shirazi, M., Brinchmann, J., \& Rahmati, A. 2014, ApJ, 787, 120

Skillman, E. D., Kennicutt, R. C., Jr. \& Hodge, P. W. 1989, ApJ, 347, 875

Skillman, E. D., Salzer, J. J., Berg, D. A., et al. 2013, AJ, 146, 3

Stasińska G. 1990, A\&AS, 83, 501

Stasińska, G., \& Izotov, Y. I. 2003, A\&A, 397, 71

Stasińska, G., Cid Fernandes, R., Mateus, A., Sodré, L., \& Asari, N. V. 2006, MNRAS, 371, 972

Thuan, T. X., Izotov, Y. I., \& Lipovetsky, V. A. 1995, ApJ, 445, 108

Thuan, T. X., Izotov, Y. I., \& Lipovetsky, V. A. 1997, ApJ, 477, 661

Thuan, T. X., Lecavelier des Etangs, A., \& Izotov, Y. I. 2005, ApJ, 621, 269

Tremonti, C. A., Heckman, T. M., Kauffmann, J., et al. 2004, ApJ, 613, 898

Whitford, A. E. 1958, AJ, 63, 201 
Table 2. Emission line intensities and equivalent widths.

\begin{tabular}{|c|c|c|c|c|c|c|}
\hline \multirow[b]{3}{*}{ Ion } & \multicolumn{6}{|c|}{ Galaxy } \\
\hline & \multicolumn{3}{|c|}{ J0143+1958 } & \multicolumn{3}{|c|}{ J0100-0028 } \\
\hline & $F(\lambda) / F(\mathrm{H} \beta)$ & $I(\lambda) / I(\mathrm{H} \beta)$ & $E W^{a}$ & $F(\lambda) / F(\mathrm{H} \beta)$ & $I(\lambda) / I(\mathrm{H} \beta)$ & $E W^{a}$ \\
\hline 3727 [O II] & $159.1 \pm 36.7$ & $141.8 \pm 39.0$ & 15.7 & $94.2 \pm 31.2$ & $106.6 \pm 37.7$ & 8.0 \\
\hline $4340 \mathrm{H} \gamma$ & $37.0 \pm 16.6$ & $46.9 \pm 25.0$ & 6.1 & $42.7 \pm 19.5$ & $46.7 \pm 24.0$ & 6.6 \\
\hline $4861 \mathrm{H} \beta$ & $100.0 \pm 25.1$ & $100.0 \pm 28.7$ & 20.7 & $100.0 \pm 30.0$ & $100.0 \pm 32.2$ & 14.9 \\
\hline 4959 [O III] & $48.6 \pm 17.4$ & $43.1 \pm 17.4$ & 8.9 & $19.0 \pm 18.9$ & $18.4 \pm 18.8$ & 1.8 \\
\hline 5007 [O III] & $139.9 \pm 31.1$ & $124.2 \pm 31.1$ & 23.3 & $54.7 \pm 22.5$ & $52.7 \pm 22.2$ & 6.6 \\
\hline $6563 \mathrm{H} \alpha$ & $318.1 \pm 62.1$ & $287.9 \pm 68.8$ & 113.8 & $343.1 \pm 79.8$ & $288.3 \pm 74.8$ & 69.7 \\
\hline 6716 [S II] & $19.1 \pm 11.2$ & $16.9 \pm 11.3$ & 5.1 & $17.8 \pm 13.4$ & $14.7 \pm 11.5$ & 4.0 \\
\hline $6731[\mathrm{~S} \mathrm{II}]$ & $9.9 \pm 10.0$ & $8.8 \pm 10.0$ & 2.7 & $21.9 \pm 14.7$ & $18.1 \pm 12.6$ & 4.3 \\
\hline 9531 [S III] & $\ldots$ & $\ldots$ & $\ldots$ & $47.8 \pm 16.5$ & $34.1 \pm 14.0$ & 29.1 \\
\hline$C(\mathrm{H} \beta)$ & & 0.005 & & & 0.205 & \\
\hline$F(\mathrm{H} \beta)^{b}$ & & 1.40 & & & 0.97 & \\
\hline \multirow[t]{3}{*}{$\mathrm{EW}(\mathrm{abs})^{a}$} & & 2.61 & & & 0.33 & \\
\hline & \multicolumn{6}{|c|}{ Galaxy } \\
\hline & \multicolumn{3}{|c|}{$\mathrm{J} 1036+2036$} & \multicolumn{3}{|c|}{ J0122+0048 } \\
\hline Ion & $F(\lambda) / F(\mathrm{H} \beta)$ & $I(\lambda) / I(\mathrm{H} \beta)$ & $E W^{a}$ & $F(\lambda) / F(\mathrm{H} \beta)$ & $I(\lambda) / I(\mathrm{H} \beta)$ & $E W^{a}$ \\
\hline 3727 [O II] & $88.2 \pm 13.5$ & $91.5 \pm 14.7$ & 31.3 & $48.7 \pm 5.7$ & $57.0 \pm 6.9$ & 29.0 \\
\hline $3869[\mathrm{Ne} \mathrm{III}]$ & $19.5 \pm 7.6$ & $20.1 \pm 7.9$ & 5.2 & $21.5 \pm 3.8$ & $24.6 \pm 4.4$ & 18.3 \\
\hline $3889 \mathrm{He} \mathrm{I}+\mathrm{H} 8$ & $\ldots$ & $\ldots$ & $\ldots$ & $23.7 \pm 3.9$ & $27.5 \pm 5.9$ & 24.2 \\
\hline $3968[\mathrm{Ne} \mathrm{III}]+\mathrm{H} 7$ & $\ldots$ & $\ldots$ & $\ldots$ & $25.4 \pm 3.9$ & $29.0 \pm 5.4$ & 31.9 \\
\hline $4101 \mathrm{H} \delta$ & $26.8 \pm 6.8$ & $28.0 \pm 8.2$ & 20.2 & $27.6 \pm 4.1$ & $30.9 \pm 5.5$ & 33.0 \\
\hline $4340 \mathrm{H} \gamma$ & $46.8 \pm 8.9$ & $48.1 \pm 10.5$ & 27.6 & $47.7 \pm 5.3$ & $51.2 \pm 6.2$ & 78.5 \\
\hline 4363 [O III] & $7.3 \pm 5.6$ & $7.4 \pm 5.7$ & 3.2 & $9.2 \pm 2.8$ & $9.8 \pm 3.0$ & 10.3 \\
\hline $4861 \mathrm{H} \beta$ & $100.0 \pm 14.1$ & $100.0 \pm 14.7$ & 78.9 & $100.0 \pm 8.8$ & $100.0 \pm 9.1$ & 147.5 \\
\hline 4959 [O III] & $72.5 \pm 11.6$ & $71.9 \pm 11.6$ & 32.5 & $86.7 \pm 7.9$ & $85.5 \pm 7.8$ & 179.6 \\
\hline 5007 [O III] & $229.9 \pm 27.5$ & $227.6 \pm 27.4$ & 91.3 & $249.8 \pm 18.5$ & $244.8 \pm 18.3$ & 468.7 \\
\hline $5876 \mathrm{He}$ I & $\ldots$ & $\ldots$ & $\ldots$ & $11.2 \pm 2.5$ & $10.0 \pm 2.3$ & 22.7 \\
\hline $6300[\mathrm{O} \mathrm{I}]$ & $\ldots$ & $\ldots$ & $\ldots$ & $1.4 \pm 1.6$ & $1.2 \pm 1.3$ & 3.2 \\
\hline 6312 [S III] & $\ldots$ & $\ldots$ & $\ldots$ & $0.6 \pm 1.5$ & $0.5 \pm 1.3$ & 1.5 \\
\hline $6563 \mathrm{H} \alpha$ & $287.8 \pm 33.2$ & $273.9 \pm 34.6$ & 238.2 & $324.5 \pm 23.4$ & $272.6 \pm 21.5$ & 1015.0 \\
\hline 6716 [S II] & $13.4 \pm 4.9$ & $12.7 \pm 4.7$ & 11.3 & $4.0 \pm 1.8$ & $3.3 \pm 1.5$ & 9.4 \\
\hline $6731[\mathrm{~S}$ II] & $10.0 \pm 4.5$ & $9.5 \pm 4.3$ & 8.3 & $3.8 \pm 1.7$ & $3.1 \pm 1.4$ & 9.0 \\
\hline 9069 [S III] & $\ldots$ & $\ldots$ & $\ldots$ & $1.8 \pm 1.1$ & $1.3 \pm 0.8$ & 17.9 \\
\hline $9531[\mathrm{~S}$ III] & $\ldots$ & $\ldots$ & $\ldots$ & $20.7 \pm 3.1$ & $14.6 \pm 2.4$ & 56.6 \\
\hline$C(\mathrm{H} \beta)$ & & 0.060 & & & 0.225 & \\
\hline$F(\mathrm{H} \beta)^{b}$ & & 3.10 & & & 8.10 & \\
\hline \multirow[t]{3}{*}{$\operatorname{EW}(\mathrm{abs})^{a}$} & & 0.44 & & & 0.41 & \\
\hline & \multicolumn{6}{|c|}{ Galaxy } \\
\hline & \multicolumn{3}{|c|}{$\mathrm{J} 0153+0104$} & \multicolumn{3}{|c|}{$\mathrm{J} 0945+3835$} \\
\hline Ion & $F(\lambda) / F(\mathrm{H} \beta)$ & $I(\lambda) / I(\mathrm{H} \beta)$ & $E W^{a}$ & $F(\lambda) / F(\mathrm{H} \beta)$ & $I(\lambda) / I(\mathrm{H} \beta)$ & $E W^{a}$ \\
\hline $3727[\mathrm{O}$ II] & $23.3 \pm 3.5$ & $25.6 \pm 4.0$ & 34.1 & $103.1 \pm 12.9$ & $129.9 \pm 17.2$ & 41.2 \\
\hline $3869[\mathrm{Ne} \mathrm{III}]$ & $38.0 \pm 4.5$ & $41.1 \pm 5.0$ & 47.0 & $35.0 \pm 6.6$ & $42.7 \pm 8.3$ & 19.7 \\
\hline $3889 \mathrm{He} \mathrm{I}+\mathrm{H} 8$ & $20.1 \pm 3.4$ & $22.4 \pm 5.5$ & 24.6 & $32.6 \pm 5.8$ & $39.7 \pm 7.6$ & 57.2 \\
\hline $3968[\mathrm{Ne}$ III] + H7 & $29.6 \pm 3.9$ & $32.2 \pm 5.2$ & 49.8 & $31.2 \pm 6.1$ & $37.5 \pm 9.3$ & 22.9 \\
\hline $4101 \mathrm{H} \delta$ & $26.7 \pm 3.6$ & $28.7 \pm 4.8$ & 48.3 & $26.9 \pm 5.5$ & $31.4 \pm 7.8$ & 25.0 \\
\hline $4340 \mathrm{H} \gamma$ & $46.5 \pm 4.9$ & $48.6 \pm 5.6$ & 98.2 & $50.0 \pm 7.7$ & $55.4 \pm 9.7$ & 42.8 \\
\hline 4363 [O III] & $14.5 \pm 2.8$ & $15.0 \pm 2.9$ & 31.2 & $10.1 \pm 3.9$ & $11.1 \pm 4.3$ & 9.4 \\
\hline $4686 \mathrm{He}$ II & $\ldots$ & $\ldots$ & & $6.3 \pm 3.7$ & $6.5 \pm 3.8$ & 4.6 \\
\hline $4861 \mathrm{H} \beta$ & $100.0 \pm 8.2$ & $100.0 \pm 8.5$ & 215.5 & $100.0 \pm 12.2$ & $100.0 \pm 12.9$ & 87.6 \\
\hline 4959 [O III] & $132.5 \pm 10.2$ & $131.1 \pm 10.2$ & 180.7 & $86.4 \pm 10.5$ & $84.7 \pm 10.4$ & 79.4 \\
\hline 5007 [O III] & $401.9 \pm 26.7$ & $396.3 \pm 26.5$ & 582.4 & $248.8 \pm 25.5$ & $241.9 \pm 25.0$ & 246.3 \\
\hline $5876 \mathrm{He} \mathrm{I}$ & $9.9 \pm 2.1$ & $9.0 \pm 2.0$ & 41.6 & $14.5 \pm 3.8$ & $12.3 \pm 3.3$ & 27.2 \\
\hline $6563 \mathrm{H} \alpha$ & $303.1 \pm 20.6$ & $272.5 \pm 20.3$ & 1432.0 & $350.1 \pm 34.5$ & $271.5 \pm 29.3$ & 440.3 \\
\hline $6716[\mathrm{~S} \mathrm{II}]$ & $1.8 \pm 1.2$ & $1.6 \pm 1.1$ & 10.0 & $\ldots$ & $\ldots$ & $\ldots$ \\
\hline $6731[\mathrm{~S} \mathrm{II}]$ & $1.8 \pm 1.2$ & $1.6 \pm 1.1$ & 9.2 & $\ldots$ & $\ldots$ & ... \\
\hline 9069 [S III] & $4.1 \pm 1.3$ & $3.4 \pm 1.2$ & 38.2 & $\ldots$ & $\ldots$ & ... \\
\hline$C(\mathrm{H} \beta)$ & & 0.135 & & & 0.330 & \\
\hline$F(\mathrm{H} \beta)^{b}$ & & 9.35 & & & 3.98 & \\
\hline $\mathrm{EW}(\mathrm{abs})^{a}$ & & 0.75 & & & 0.22 & \\
\hline
\end{tabular}

Notes. ${ }^{(a)}$ In angstroms. ${ }^{(b)}$ In units of $10^{-16} \mathrm{erg} \mathrm{s}^{-1} \mathrm{~cm}^{-2}$. 
Table 2. continued.

\begin{tabular}{|c|c|c|c|c|c|c|}
\hline \multirow[b]{3}{*}{ Ion } & \multicolumn{6}{|c|}{ Galaxy } \\
\hline & \multicolumn{3}{|c|}{ J1228-0125 } & \multicolumn{3}{|c|}{ J0222-0935 } \\
\hline & $F(\lambda) / F(\mathrm{H} \beta)$ & $I(\lambda) / I(\mathrm{H} \beta)$ & $E W^{a}$ & $F(\lambda) / F(\mathrm{H} \beta)$ & $I(\lambda) / I(\mathrm{H} \beta)$ & $E W^{a}$ \\
\hline 3727 [O II] & $130.1 \pm 22.7$ & $127.5 \pm 23.9$ & 23.3 & $109.9 \pm 17.4$ & $108.1 \pm 19.3$ & 83.5 \\
\hline $3869[\mathrm{Ne} \mathrm{III}]$ & $12.6 \pm 9.9$ & $12.3 \pm 9.9$ & 2.8 & $24.3 \pm 7.7$ & $23.8 \pm 8.1$ & 15.7 \\
\hline $3889 \mathrm{He} \mathrm{I}+\mathrm{H} 8$ & $\ldots$ & $\ldots$ & $\ldots$ & $15.1 \pm 6.1$ & $17.8 \pm 7.9$ & 16.7 \\
\hline $3968[\mathrm{Ne}$ III $]+\mathrm{H} 7$ & $\ldots$ & $\ldots$ & $\ldots$ & $16.4 \pm 7.2$ & $23.3 \pm 11.7$ & 7.5 \\
\hline $4101 \mathrm{H} \delta$ & $30.0 \pm 10.5$ & $35.5 \pm 13.9$ & 9.6 & $16.0 \pm 6.6$ & $20.4 \pm 9.4$ & 10.8 \\
\hline $4340 \mathrm{H} \gamma$ & $39.9 \pm 11.3$ & $44.7 \pm 13.8$ & 14.1 & $39.9 \pm 9.3$ & $42.9 \pm 10.9$ & 28.0 \\
\hline 4363 [O III] & $\ldots$ & $\ldots$ & $\ldots$ & $10.0 \pm 6.4$ & $9.6 \pm 6.5$ & 4.8 \\
\hline $4861 \mathrm{H} \beta$ & $100.0 \pm 17.5$ & $100.0 \pm 17.9$ & 99.8 & $100.0 \pm 16.3$ & $100.0 \pm 17.6$ & 53.4 \\
\hline 4959 [O III] & $56.8 \pm 12.9$ & $55.6 \pm 12.9$ & 22.1 & $86.0 \pm 14.6$ & $80.5 \pm 14.6$ & 50.6 \\
\hline 5007 [O III] & $169.3 \pm 26.4$ & $166.0 \pm 26.4$ & 65.1 & $248.3 \pm 33.8$ & $232.1 \pm 33.7$ & 73.7 \\
\hline $6563 \mathrm{H} \alpha$ & $264.7 \pm 37.8$ & $261.0 \pm 41.3$ & 340.8 & $299.7 \pm 39.4$ & $271.1 \pm 41.3$ & 284.3 \\
\hline $6716[\mathrm{~S} \mathrm{II}]$ & $\ldots$ & $\ldots$ & $\ldots$ & $23.6 \pm 6.7$ & $21.0 \pm 6.5$ & 22.6 \\
\hline 6731 [S II] & $\ldots$ & $\ldots$ & $\ldots$ & $12.5 \pm 5.4$ & $11.1 \pm 5.2$ & 10.2 \\
\hline$C(\mathrm{H} \beta)$ & & 0.000 & & & 0.065 & \\
\hline$F(\mathrm{H} \beta)^{b}$ & & 2.08 & & & 2.41 & \\
\hline $\operatorname{EW}(\mathrm{abs})^{a}$ & & 2.00 & & & 3.45 & \\
\hline
\end{tabular}

Table 3. Ionic and total heavy element abundances ( $T_{\mathrm{e}}$-method).

\begin{tabular}{|c|c|c|c|c|c|}
\hline & & & Galaxy & & \\
\hline Property & $\mathrm{J} 1036+2036$ & $\mathrm{~J} 0122+0048$ & J0153+0104 & $\mathrm{J} 0945+3835$ & J0222-0935 \\
\hline$\overline{T_{\mathrm{e}}(\mathrm{O} \text { III })(\mathrm{K})}$ & $19694 \pm 9122$ & $22129 \pm 4638$ & $21544 \pm 2824$ & $24350 \pm 7071$ & $22604 \pm 10766$ \\
\hline$T_{\mathrm{e}}(\mathrm{O}$ II $)(\mathrm{K})$ & $15583 \pm 6684$ & $15540 \pm 2928$ & $15604 \pm 1859$ & $14985 \pm 3595$ & $15462 \pm 6547$ \\
\hline $\mathrm{O}^{+} / \mathrm{H}^{+}\left(\times 10^{4}\right)$ & $0.074 \pm 0.081$ & $0.049 \pm 0.024$ & $0.022 \pm 0.007$ & $0.118 \pm 0.076$ & $0.089 \pm 0.097$ \\
\hline $\mathrm{O}^{++} / \mathrm{H}^{+}\left(\times 10^{4}\right)$ & $0.132 \pm 0.140$ & $0.116 \pm 0.053$ & $0.195 \pm 0.057$ & $0.096 \pm 0.059$ & $0.105 \pm 0.109$ \\
\hline $\mathrm{O} / \mathrm{H}\left(\times 10^{4}\right)$ & $0.207 \pm 0.162$ & $0.165 \pm 0.058$ & $0.217 \pm 0.057$ & $0.214 \pm 0.096$ & $0.195 \pm 0.146$ \\
\hline $12+\log (\mathrm{O} / \mathrm{H})$ & $7.316 \pm 0.340$ & $7.216 \pm 0.154$ & $7.336 \pm 0.115$ & $7.330 \pm 0.195$ & $7.289 \pm 0.325$ \\
\hline $\mathrm{Ne}^{++} / \mathrm{H}^{+}\left(\times 10^{5}\right)$ & $0.264 \pm 0.279$ & $0.249 \pm 0.111$ & $0.440 \pm 0.126$ & $0.354 \pm 0.198$ & $0.230 \pm 0.223$ \\
\hline ICF & 1.153 & 1.125 & 1.043 & 1.243 & 1.198 \\
\hline $\log (\mathrm{Ne} / \mathrm{O})$ & $-0.833 \pm 0.709$ & $-0.769 \pm 0.288$ & $-0.674 \pm 0.176$ & $-0.686 \pm 0.479$ & $-0.849 \pm 0.727$ \\
\hline
\end{tabular}


N. G. Guseva et al.: New XMD galaxies in SDSS/BOSS DR10

Table 4. Ionic and total heavy element abundances (strong-line method).

\begin{tabular}{|c|c|c|c|c|}
\hline & \multicolumn{4}{|c|}{ Galaxy } \\
\hline Property & $\mathrm{J} 0143+1958$ & J0100-0028 & $\mathrm{J} 1036+2036$ & $\mathrm{~J} 0122+0048$ \\
\hline$T_{\mathrm{e}}(\mathrm{O}$ III $)(\mathrm{K})$ & $19549 \pm 1016$ & $22841 \pm 1592$ & $18307 \pm 516$ & $18214 \pm 323$ \\
\hline$T_{\mathrm{e}}(\mathrm{O}$ II $)(\mathrm{K})$ & $15567 \pm 1278$ & $15416 \pm 2119$ & $15344 \pm 635$ & $15321 \pm 397$ \\
\hline $\mathrm{O}^{+} / \mathrm{H}^{+}\left(\times 10^{4}\right)$ & $0.115 \pm 0.039$ & $0.102 \pm 0.051$ & $0.073 \pm 0.014$ & $0.049 \pm 0.007$ \\
\hline $\mathrm{O}^{++} / \mathrm{H}^{+}\left(\times 10^{4}\right)$ & $0.075 \pm 0.018$ & $0.023 \pm 0.010$ & $0.152 \pm 0.018$ & $0.174 \pm 0.013$ \\
\hline $\mathrm{O} / \mathrm{H}\left(\times 10^{4}\right)$ & $0.190 \pm 0.044$ & $0.126 \pm 0.052$ & $0.226 \pm 0.023$ & $0.222 \pm 0.014$ \\
\hline $12+\log (\mathrm{O} / \mathrm{H})$ & $7.279 \pm 0.100$ & $7.100 \pm 0.179$ & $7.354 \pm 0.045$ & $7.347 \pm 0.028$ \\
\hline $\mathrm{Ne}^{++} / \mathrm{H}^{+}\left(\times 10^{5}\right)$ & $\ldots$ & $\ldots$ & $0.297 \pm 0.120$ & $0.374 \pm 0.069$ \\
\hline ICF & $\ldots$ & $\ldots$ & 1.138 & 1.092 \\
\hline \multirow[t]{2}{*}{$\log (\mathrm{Ne} / \mathrm{O})$} & $\ldots$ & $\cdots$ & $-0.825 \pm 0.233$ & $-0.736 \pm 0.098$ \\
\hline & \multicolumn{4}{|c|}{ Galaxy } \\
\hline Property & $\mathrm{J} 0153+0104$ & J0945+3835 & $\mathrm{J} 1228-0125$ & J0222-0935 \\
\hline$T_{\mathrm{e}}(\mathrm{O}$ III $)(\mathrm{K})$ & $16067 \pm 308$ & $17307 \pm 417$ & $18827 \pm 645$ & $17690 \pm 579$ \\
\hline$T_{\mathrm{e}}(\mathrm{O}$ II $)(\mathrm{K})$ & $14553 \pm 366$ & $15052 \pm 506$ & $15456 \pm 802$ & $15176 \pm 707$ \\
\hline $\mathrm{O}^{+} / \mathrm{H}^{+}\left(\times 10^{4}\right)$ & $0.026 \pm 0.004$ & $0.110 \pm 0.017$ & $0.106 \pm 0.024$ & $0.091 \pm 0.019$ \\
\hline $\mathrm{O}^{++} / \mathrm{H}^{+}\left(\times 10^{4}\right)$ & $0.370 \pm 0.027$ & $0.192 \pm 0.019$ & $0.108 \pm 0.017$ & $0.177 \pm 0.025$ \\
\hline $\mathrm{O} / \mathrm{H}\left(\times 10^{4}\right)$ & $0.396 \pm 0.027$ & $0.302 \pm 0.026$ & $0.213 \pm 0.029$ & $0.269 \pm 0.032$ \\
\hline $12+\log (\mathrm{O} / \mathrm{H})$ & $7.598 \pm 0.030$ & $7.480 \pm 0.038$ & $7.329 \pm 0.060$ & $7.429 \pm 0.051$ \\
\hline $\mathrm{Ne}^{++} / \mathrm{H}^{+}\left(\times 10^{5}\right)$ & $0.868 \pm 0.112$ & $0.732 \pm 0.148$ & $0.180 \pm 0.145$ & $0.395 \pm 0.135$ \\
\hline ICF & 1.026 & 1.156 & 1.215 & 1.145 \\
\hline $\log (\mathrm{Ne} / \mathrm{O})$ & $-0.648 \pm 0.066$ & $-0.552 \pm 0.125$ & $-0.989 \pm 0.573$ & $-0.774 \pm 0.204$ \\
\hline
\end{tabular}

